\title{
Brillouin Scattering from Smectic Liquid Crystals
}

\section{Citation}

Liao, York, Noel A. Clark, and Peter S. Pershan. 1973. Brillouin scattering from smectic liquid crystals. Physical Review Letters 30(14): 639-641.

\section{Published Version}

doi:10.1103/PhysRevLett.30.639

\section{Permanent link}

http://nrs.harvard.edu/urn-3:HUL.InstRepos:10361963

\section{Terms of Use}

This article was downloaded from Harvard University's DASH repository, and is made available under the terms and conditions applicable to Other Posted Material, as set forth at http:// nrs.harvard.edu/urn-3:HUL.InstRepos:dash.current.terms-of-use\#LAA

\section{Share Your Story}

The Harvard community has made this article openly available.

Please share how this access benefits you. Submit a story.

\section{Accessibility}




\title{
Brillouin Scattering from Smectic Liquid Crystals*
}

\author{
York Liao, Noel A. Clark, and P. S. Pershan \\ Division of Engineering and Applied Physics, Havvard University, Cambridge, Massachusetts 02138
}

(Received 2 January 1973)

\begin{abstract}
Brillouin-scattering results are reported for monodomain samples of $\beta$-methyl butyl $p[(p$-methoxy benzylidine)amino $]$ cinnamate in both the smectic- $A$ and $-B$ phases. For both phases theoretical predictions are confirmed concerning the number and polarizations of the propagating hypersonic waves. We report quantitative measurements of various thermodynamic derivatives, including the coupling between density and smectic-layer spacing. We discuss preliminary evidence concerning one model of the smectic- $B$ phase.
\end{abstract}

Qualitative differences between the elastic properties of various liquid crystal phases, and between those of liquid crystals and crystals, provide one practical scheme by which the different phases can be classified. ${ }^{1}$ Although classification in terms of the types of long-range order may be more fundamental, the two schemes can be connected with the aid of a phenomenological elastohydrodynamic theory. ${ }^{2,3}$ We present here Brillouin-scattering results on the speed and polarization of propagating hypersonic waves in monodomain samples of the smectic- $A$ and uniaxial smectic- $B$ phases of $\beta$-methyl butyl $p[(p-$ methoxy benzylidine)amino] cinnamate. ${ }^{4}$ The results are interpreted in terms of a phenomenological elastic-hydrodynamic theory and the differences between the various phases discussed. ${ }^{2}$

We only sketch the most important experimental features since a more complete description will be given elsewhere. Samples were placed between lecithin-treated glass slides separated by $250 \mu \mathrm{m}$ and heated $\left(T \geqslant 99.6^{\circ} \mathrm{C}\right)$ to the isotropic phase. Upon slow cooling we obtained successively samples in the nematic $\left(99.6^{\circ} \mathrm{C} \gtrsim T \gtrsim 81.0^{\circ} \mathrm{C}\right)$, monodomain smectic $-A\left(81.0^{\circ} \mathrm{C} \gtrsim T \gtrsim 59.8^{\circ} \mathrm{C}\right)$, and monodomain uniaxial $B\left(59.8^{\circ} \mathrm{C} \gtrsim T\right)$ phases. ${ }^{4} \mathrm{Be}-$ low $47.0^{\circ} \mathrm{C}$ the smectic $-B$ phase was actually supercooled since slight disturbance would cause it to suddenly become polycrystalline. Microscopic observations confirmed the uniaxial monodomain character of the samples and were used, along with calorimetry, to determine the transition temperatures.

In the experiment a collimated beam of linearly polarized 5145 - $\AA$ light from a single-mode argonion laser was incident on the sample with wave vector $\overrightarrow{\mathrm{k}}_{i}$. To avoid excess absorption and photochemically induced deterioration of the sample, the incident power was kept below $200 \mathrm{~mW}$. Light of a selected polarization, scattered through a selected angle $\theta$, of wave vector $\vec{k}_{s}$, was collect- ed and frequency analyzed using a double-pass flat Fabry-Perot interferometer. ${ }^{5}$ This geometry allowed for the study of elastic modes having selected wave vector $\overrightarrow{\mathrm{q}}=\overrightarrow{\mathrm{k}}_{s}-\overrightarrow{\mathrm{k}}_{i}$ and polarization.

When both incident and scattered light are polarized as extraordinary waves $(E \rightarrow E)$, we easily observed two underdamped resonances that could be identified as propagating waves [for example, see Figs. 1(a) and 1(b)]. The magnitude of $\vec{q}$ was varied by slightly more than a factor of $2(1.4 \lesssim \lambda q / 2 \pi \lesssim 3.2, \lambda=5145 \AA)$, and in all cases the ratio of the observed frequency shifts $\Delta \omega$ to $q(\Delta \omega / q$ is the speed of propagation) was independent of $q$ within experimental error.

In the hydrodynamic approximation the speed and polarization of the propagating acoustic waves of a liquid crystal can be completely described by the same formalism as is conventionally em-
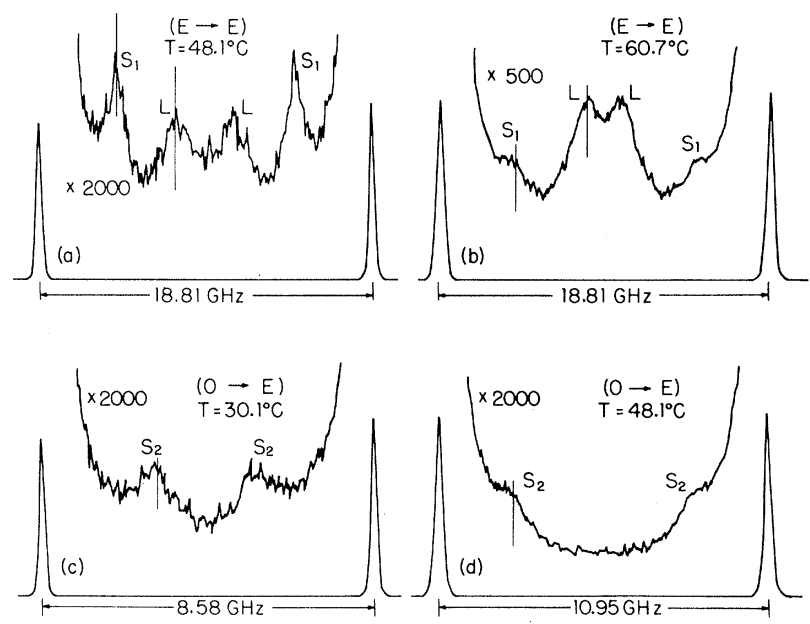

FIG. 1. Typical Brillouin spectra for $\theta=150^{\circ}$ showing longitudinal $(L)$, first shear $\left(s_{1}\right)$, and second shear $\left(s_{2}\right)$ modes. (a) Smectic $B, \psi=38.1^{\circ}$; (b) smectic $A$, $\psi$ $=46.9^{\circ}$; (c) supercooled smectic $B, \psi=43.0^{\circ}$; (d) smec$\operatorname{tic} B, \psi=48.0^{\circ}$. 
ployed in the discussion of crystals. ${ }^{6}$ The relevant elastic tensor of the smectic- $A$ phase is the same as that of an hexagonal crystal except that $C_{11}=C_{12}$ and $C_{44}=0$. This predicts only two propagating waves with speeds $v_{1}(\psi)$ and $v_{3}(\psi),{ }^{7}$

$$
\begin{aligned}
& v_{1}^{2}+v_{3}^{2}=\rho^{-1}\left[C_{11}+\left(C_{33}-C_{11}\right) \cos ^{2} \psi\right], \\
& v_{1}^{2} v_{3}^{2}=\rho^{-2}\left[C_{11} C_{33}-C_{13}^{2}\right] \cos ^{2} \psi \sin ^{2} \psi,
\end{aligned}
$$

where $\rho$ is the mass density and $\psi$ is the angle between $\vec{q}$ and the uniaxial direction (the $z$ axis). Figure 2(a) is a polar plot of the speeds $v_{1}(\psi)$ and $v_{3}(\psi)$ obtained for both the $(0-0)$ and $(E \rightarrow E)$ observations on the smectic- $A$ phase together with the angular dependence calculated from Eqs. (1) choosing values of $C_{11}, C_{33}$, and $\left|C_{13}\right|$ that obtains the best fit. The positive sign $\left(C_{13}>0\right)$ is the only choice that makes physical sense. The actual values for the $C_{i j}$ for various temperatures are contained in Fig. 3. The "shearlike" mode $\left(v_{1}\right)$ appeared to become weaker as $\psi$ approached $45^{\circ}$ and could not be observed for angles greater than those shown in Fig. 1(a). This would be the predicted behavior if the intensity of the shear mode was dominated by a flow alignment

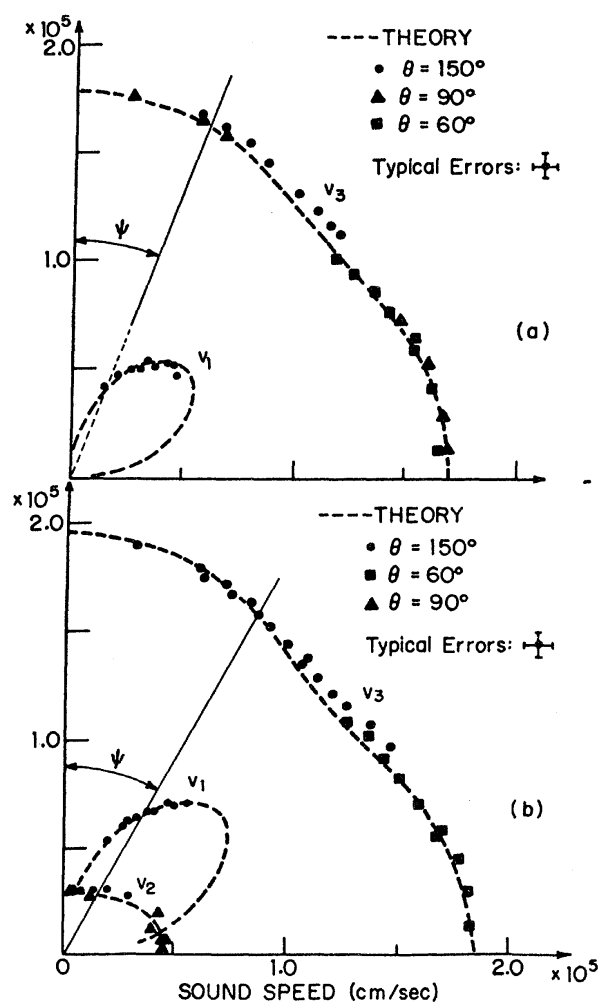

FIG. 2. Dependence of measured and calculated sound speeds on polar angle $\psi$. (a) Smectic $A\left(T=60.7^{\circ} \mathrm{C}\right)$; (b) smectic $B\left(T=48 \cdot 1^{\circ} \mathrm{C}\right)$. process; $\delta \epsilon_{x z} \sim \nabla_{z} v_{x}$. Further experiments will be necessary to provide a more definite interpretation.

We have also searched for a third propagating mode using depolarized light scattering $(E \rightarrow O$ and $O \rightarrow E$ ). Although we have observed such a mode in the smectic- $B$ phase, we could not detect one in the $A$ phase [see, for example, Figs. 1(c) and $1(d)$ ]. Since the general expression for the speed of this wave in an hexagonal crystal is

$$
v_{2}^{2}(\psi)=\left(C_{44} / \rho\right) \cos ^{2} \psi+\left[\frac{1}{2}\left(C_{11}-C_{12}\right) \rho\right] \sin ^{2} \psi,
$$

the present experiments place upper limits on $C_{44}$ and $\frac{1}{2}\left(C_{11}-C_{12}\right)$ to be less than $\sim 3 \times 10^{-3} C_{33}$ for the smectic- $A$ phase. Other experiments on the intensity distribution of light by static defects in these samples actually place a smaller upper limit of $C_{44} / C_{33} \lesssim 10^{-4}$. $^{8}$

Figure 2(b) shows the hypersonic speeds versus $\psi$ for the smectic- $B$ phase. Two of the modes, $v_{1}(\psi)$ and $v_{3}(\psi)$, are observed in the polarized spectra $(E-E)$ and the third $v_{2}(\psi)$ is observed in depolarized spectra $(E \rightarrow O$ or $O \rightarrow E)$. The existence of this third wave follows from the crystal-like ordering within the smectic layers, which explains the observation that $\left(C_{11}-C_{12}\right) C_{11}{ }^{-1} \approx 0.1$

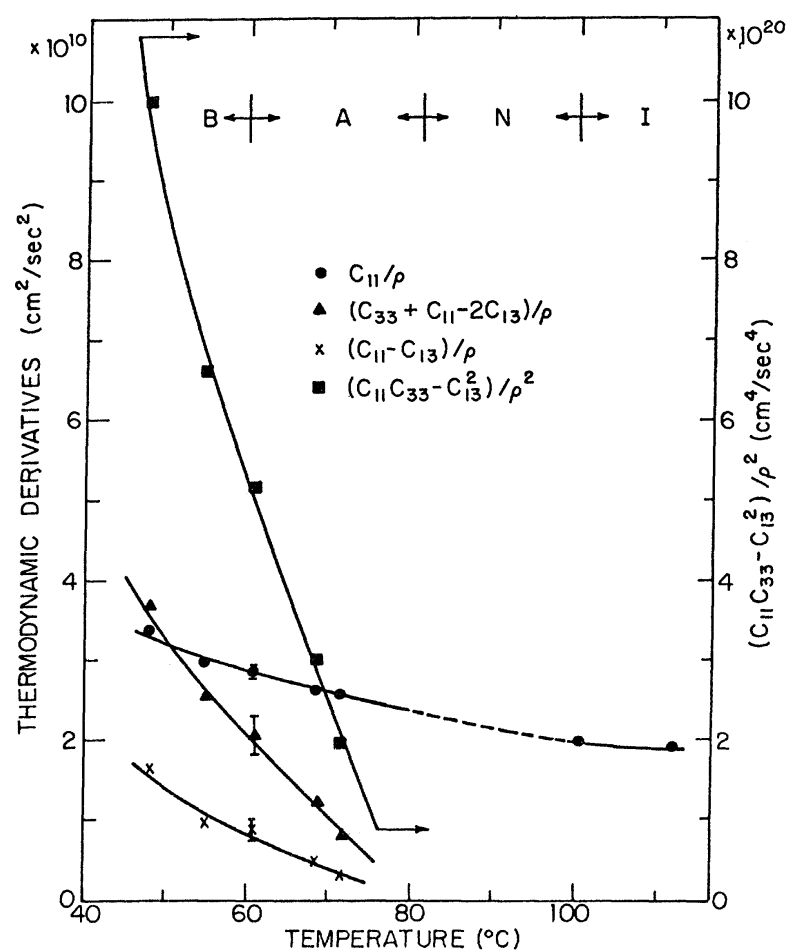

FIG. 3. Temperature dependence of various combinations of the first-order elastic constants of smectic $A$ and $B$. 
$\neq 0$. The ratio does not appear to have a significant temperature dependence. One possible difference, that has been suggested, between the smectic $-B$ and the crystalline phase is that $C_{44}$ $=0$ in the $B$ and not in the crystal. ${ }^{9}$ Comparison between the data and Eq. (2) yields $C_{44} / C_{11} \approx 0.02$ $\neq 0$ with no significant temperature dependence. This value agrees with estimates obtained from analysis of a separate experiment on the intensity distribution of light scattered by static defects. $^{8}$ In spite of this evidence, given the importance of the question, we believe it would be prudent to reserve final judgment pending further experiments. For example, the apparent finite value could result from relaxation effects that cause the effective $C_{44}(\omega, q)$ at Brillouin frequencies and wavelengths to be different from the value at $(\omega, q) \rightarrow 0$.

In principle, with a finite value for $C_{44}$, Eq. (1) must be modified to properly describe the speeds of the two other elastic waves in the smectic- $B$ phase. In practice, the observed value of $C_{44}$ is sufficiently small that the errors resulting from neglecting the modifications are smaller than the experimental uncertainties. Thus, we also fit the two modes observed in polarized $(E \rightarrow E)$ scattering from the $B$ phase to Eq. (1).

In the hydrodynamic regime, $(\omega, q) \rightarrow 0$, with $C_{44}=0$, the following thermodynamic derivatives can be related to the elastic constants for smec$\operatorname{tic} A:\left(\partial \varphi_{3} / \partial \nabla_{3} x\right)_{s, \rho}=C_{33}+C_{11}-2 C_{13},\left(\partial P / \partial \nabla_{3} x\right)_{s, \rho}$ $=\rho\left(\partial \varphi_{3} / \partial \rho\right)_{x, s}=C_{11}-C_{13}, \rho(\partial P / \partial \rho)_{s, x}=C_{11} \cdot{ }^{10}$ In Fig. 3 we plot the temperature dependence of the above combinations. Although the compressibility $\left(C_{11}^{-1}\right)$ has only weak temperature dependence that appears to vary continuously across the different phases, the other combinations of elastic constants vary strongly with temperature, extrapolating towards zero as $T^{*}-T$ at the nematicsmectic- $A$ transition temperature $T^{*}$. This is exactly the behavior predicted by the de Gennes analogy to the superfluid when one includes the coupling required between the smectic layers and the density to obtain $C_{11} \neq C_{13} \cdot{ }^{11}$ Unfortunately, in its simplest modification that theory predicts a second-order phase transition between the nematic and smectic phases, and we observe a latent heat. ${ }^{4}$ If we follow McMillan, ${ }^{12}$ de Gennes's theory can be modified to predict a first-order transition by introducing coupling between the smectic and nematic order parameters. The linear temperature dependence could then be understood in terms of saturation of the nematic ordering. The requirement of stability $C_{11} C_{33}-C_{13}{ }^{2}$
$>0$ provides one test of the calculated elastic constants. $^{2}$ Figure 3 shows the temperature dependence of this quantity, and it is positive.

To summarize, the most important results of this work are (i) confirmation, for both smectics $A$ and $B$, of the predictions concerning the number of propagating hypersonic waves and their polarization; (ii) the first measure of the thermodynamic derivative that describes the coupling between density and layer spacing in smectics $\left(C_{11}-C_{13} \neq 0\right)^{13}$; in addition, for the smectic $B$ we have observed (iii) an apparent $C_{44} \neq 0$. This must be examined more carefully by both the present technique and others since if $C_{44}(\omega, q)$ remains finite as $(\omega, q) \rightarrow 0$, it eliminates one suggested model for the smectic- $B$ phase. ${ }^{9}$

We are indebted to Dr. Donald O'Shea for initial collaboration on the construction of our Fabry-Perot interferometer, and to Professor Robert B. Meyer and Professor David Dolphin for discussions and technical advice.

*Work supported in part by the National Science Foundation under Grant No. GH-34401, and by the Division of Engineering and Applied Physics, Harvard University.

${ }^{1}$ Other approaches to classification of liquid crystals are discussed in H. Sackmann and D. Demus, Mol. Cryst. $\underline{2}, 81$ (1966); S. Diele, P. Brand, and H. Sackmann, Mol. Cryst. 16, 105 (1972).

${ }^{2}$ P. C. Martin, O. Parodi, and P. S. Pershan, Phys. Rev. A $\underline{6}, 2401$ (1972).

${ }^{3}$ F. Jähnig and H. Schmidt, Ann. Phys. (New York) 71, 129 (1972).

${ }^{4}$ M. Leciercq, J. Billard, and J. Jacques, Mol. Cryst. 8. 367 (1969). This identification of the phases was made on the basis of the above paper and our own microscopic, calorimeteric, and $\mathrm{x}$-ray studies.

${ }^{5} \mathrm{~J}$. R. Sandercock, Opt. Commun. 2, 73 (1970).

${ }^{6} \mathrm{H}$. B. Huntington, in Solid State Physics, edited by F. Seitz and D. Turnbull (Academic, New York, 1958), Vol 7, p. 213. See Sect. I.3.b., p. 222.

${ }^{7}$ P. G. de Gennes, J. Phys. (Paris), Colloq. 30, C465 (1969).

${ }^{8}$ These are preliminary measurements using the techniques described in N. Clark and P. S. Pershan, Phys. Rev. Lett. 30,3 (1973).

${ }^{9}$ See Sect. V of Ref. 2 and also P. G. de Gennes and G. Sarma, Phys. Lett. 38A, 219 (1972).

${ }^{10} \mathrm{As}$ discussed more fully in Ref. $2, \phi_{3}$ is the thermodynamic force conjugate to the strain of the smectic layers $\left(\delta a / a=\nabla_{3} x\right)$ and $P$ is the pressure. In smectic $B$ the same combination of elastic constants would involve more thermodynamic derivatives; for example, $\partial \phi_{33} / \partial \nabla_{1} x_{1}, \partial \phi_{11} / \partial \nabla_{1} x_{1}, \partial \phi_{11} / \partial \rho$, etc.

${ }^{11}$ P. G. de Gennes, Solid State Commun. 10, 753 (1972).

${ }^{12}$ W. L. McMillan, Phys. Rev. A 4, 1238 (1971).

${ }^{13}$ The first measurement that $C_{33} \neq C_{11}$ was reported by A. E. Lord, Jr., Phys. Rev. Lett. 29, 1366 (1972). 